\title{
Phase retrieval for solutions of the Shrödinger and Helmholtz equations
}

\author{
Philippe JAMING \\ Univ. Bordeaux, IMB, UMR 5251 \\ F-33400 Talence, France \\ CNRS, IMB, UMR 5251 \\ F-33400 Talence, France \\ Philippe.Jaming@math.u-bordeaux.fr
}

\begin{abstract}
In this paper we give an overview of our recent results on the phase retrieval problem for solutions of two partial differential equations: the free Shrödinger equation and the Helmholtz equation. In particular, we show that structured signals often imply uniqueness in the phase retrieval problem.

Phase retrieval;Shrödinger equation; Helmholtz equation
\end{abstract}

\section{INTRODUCTION}

The phase retrieval problem consists in reconstructing a function from its modulus or the modulus of some transform of it (frame coefficient, Fourier transform,...) and some structural information on the function (e.g. to be compactly supported). Such a problem occurs in many scientific fields: microscopy, holography, crystallography, neutron radiography, optical coherence tomography, optical design, radar signal processing, quantum mechanics to name a few. We refer to the books $[\mathrm{Hu}],[\mathrm{St}]$, the review articles [KST], [Mi], [Fi], [LBL] and to the introduction of our previous paper [Ja] for descriptions of various instances of this problems, for some solutions to it (both theoretical and numerical) and for further references.

The problem can be split into two main questions:

- design algorithms for the reconstruction of at least one solution.

- obtain uniqueness results.

After having long been ignored by mathematicians, recent progress on the algorithmic aspect of the problem [CSV], [WdAM] has triggered a lot of attention to this problem. While the design of numerical algorithms that allow the reconstruction of one solution is of course essential, the task can only be complete once one is certain to reconstruct all solutions of interest. This is generally not possible as long as uniqueness is not guarantied and plainly justifies the second part of the problem. Uniqueness is also usefull in order to have stability results in presence of additive noise. In this paper, we will only deal with the uniqueness aspect of the problem. More precisely, the phase retrieval problem is extremely common in optical sciences. Among other reasons, this is due to the lack of sensitivity of optical measurement instruments to phase. It turns out that optical signals are solution of Partial Differential Equations and our aim is to show that this information can be of some use in the phase retrieval problem. We will concentrate on two equations.
The first equation we consider is the free Shrödinger equation on $\mathbb{R}^{d}$ with initial condition $u_{0} \in L^{2}\left(\mathbb{R}^{d}\right)$ :

$$
\left\{\begin{array}{l}
i \partial_{t} u+\frac{1}{4 \pi} \Delta_{x}^{2} u=0 \\
u(x, 0)=u_{0}(x)
\end{array}\right.
$$

where $\Delta_{x}=\sum_{j} \partial_{x_{j}}^{2}$ is the usual Laplace operator. In this case, we ask the following:

Problem 1 (Phase Retrieval Problem for the Free Shrödinger Equation).

Let $u_{0}, v_{0} \in L^{2}\left(\mathbb{R}^{d}\right)$ and let $u$ and $v$ be the solutions of the Free Shrödinger equation (I.1) with initial value $u_{0}$ and $v_{0}$. Let $\tau=\left\{t_{i}\right\}_{i \in I} \subset[0,+\infty)$ be a set of times (finite or not) at which one measures $|u|$ and $|v|$. Assume that

$$
\left|u\left(x, t_{i}\right)\right|=\left|v\left(x, t_{i}\right)\right| \quad \text { for all } i \in I .
$$

i) Does this imply that $u=c v$ for some constant $c \in \mathbb{C}$, $|c|=1$ ?

ii) If we restrict $u \in \mathcal{D}$ for some set $\mathcal{D} \subset L^{2}\left(\mathbb{R}^{d}\right)$ does (I.2) imply that $u=c v$ for some constant $c \in \mathbb{C},|c|=1$ ?

iii) If we further restrict both $u, v \in \mathcal{D}$ for some set $\mathcal{D} \subset$ $L^{2}\left(\mathbb{R}^{d}\right)$ does (I.2) imply that $u=c v$ for some constant $c \in \mathbb{C},|c|=1$ ?

The second equation we consider is the Helmholtz equation on $\mathbb{R}^{d}$

$$
\Delta u+u=0 .
$$

An entire (defined on the whole of $\mathbb{R}^{d}$ ) solution $u$ of (I.3) can be expressed in polar coordinates as a series

$$
u(r \theta) \sim \frac{(2 \pi)^{1 / 2}}{r^{(d-2) / 2}} \sum_{m=0}^{\infty} \sum_{j=1}^{N(m)} a_{m, j} J_{\nu(m)}(r) Y_{m}^{j}(\theta)
$$

where $J_{\nu}$ are the Bessel functions, $\nu(m)=m+(d-2) / 2$ and $\left\{Y_{m}^{j}\right\}_{j=1, \ldots, N(m)}$ is a basis for the spherical harmonics of degree $m$ in $\mathbb{R}^{d}$.

The far-field-pattern $f$ is defined to be the spherical part of $r^{(d-1) / 2} u(r \theta)$ as $r \rightarrow \infty$, i.e., for $\theta \in \mathbb{S}^{d-1}$ (the unit sphere of $\mathbb{R}^{d}$ ),

$$
f(\theta) \sim \sum_{m=0}^{\infty} \sum_{j=1}^{N(m)} a_{m, j}(f) Y_{m}^{j}(\theta) .
$$


A solution $u$ of (I.4) is said to be a Herglotz wave function if its far-field-pattern is a function $f \in L^{2}\left(\mathbb{S}^{d-1}\right)$ and we then write $u=u_{f}$. We will denote the space of Herglotz wave functions by $\mathcal{W}^{2}\left(\mathbb{R}^{d}\right)$. The problem we then address is the following:

Problem 2 (Phase Retrieval Problem for the Helmholtz Equation).

Let $u_{f}, u_{g} \in \mathcal{W}^{2}\left(\mathbb{R}^{d}\right)$ be such that $\left|u_{g}\right|=\left|u_{f}\right|$. Does this imply that either $u_{g}=c u_{f}$ or $u_{g}=c \bar{u}_{f}$ for some $c \in \mathbb{C}$ with $|c|=1$.

What if $f$ is in some subspace of $L^{2}\left(\mathbb{S}^{d-1}\right)$, or if both $f, g$ belong to some subspace $L^{2}\left(\mathbb{S}^{d-1}\right)$.

In this paper, we will report on our work $[\mathrm{Ja}],[\mathrm{AJ}],[\mathrm{JPE}]$ to show that, under some structural restriction on the solution, uniqueness may be achieved in the phase retrieval problem.

\section{THE FREE SHRÖDINGER EQUATION}

\section{A. Reformulation of the problem}

Let us recall that the solution of the Free Shrödinger Equation (I.1) with initial data $u_{0} \in L^{2}(\mathbb{R})$ has solution

$u(x, t)=\int_{\mathbb{R}} e^{-i \pi|\xi|^{2} t+2 i \pi\langle x, \xi\rangle} \widehat{u_{0}}(\xi) \mathrm{d} \xi=\mathcal{F}^{-1}\left[e^{-i \pi \xi^{2} t} \widehat{u_{0}}\right](x)$

where $\mathcal{F}^{-1}$ is the inverse Fourier transform. Let us introduce the Fractional Fourier Transform (FrFT): let $\alpha \in \mathbb{R} \backslash \pi \mathbb{Z}$, let $c_{\alpha}=\frac{\exp \frac{i}{2}\left(\alpha-\frac{\pi}{2}\right)}{\sqrt{|\sin \alpha|}}$. For $u \in L^{1}\left(\mathbb{R}^{d}\right)$ and $\alpha \notin \pi \mathbb{Z}$, define

$$
\begin{aligned}
\mathcal{F}_{\alpha} u(\xi)= & c_{\alpha} e^{-i \pi|\xi|^{2} \cot \alpha} \times \\
& \times \int_{\mathbb{R}} u(t) e^{-i \pi t^{2} \cot \alpha} e^{-2 i \pi t \xi / \sin \alpha} \mathrm{d} t .
\end{aligned}
$$

A straightforward computation then shows that, for $\alpha \in$ $(-\pi / 2, \pi / 2)$,

$$
\mathcal{F}_{\alpha} u_{0}(\xi)=\left(\frac{i e^{i \alpha / 2}}{\sqrt{|\cos \alpha|}}\right)^{d} e^{-i \pi|\xi|^{2} \cot \alpha} u(\xi / \cos \alpha, \cot \alpha) .
$$

Next, let us now recall that the ambiguity function of $u \in$ $L^{2}(\mathbb{R})$ is defined by

$$
A(u)(x, y)=\int_{\mathbb{R}} u\left(t+\frac{x}{2}\right) \overline{u\left(t-\frac{x}{2}\right)} e^{-2 i \pi t y} \mathrm{~d} t .
$$

The link between the FrFT and the ambiguity function comes from the following property:

$$
\begin{aligned}
& A\left(\mathcal{F}_{\alpha} u\right)(x, y) \\
& \quad=A(u)(x \cos \alpha-y \sin \alpha, x \sin \alpha+y \cos \alpha) .
\end{aligned}
$$

In particular,

$$
\begin{aligned}
A(u)(-y \sin \alpha, y \cos \alpha) & =A\left(\mathcal{F}_{\alpha} u\right)(0, y) \\
& =\mathcal{F}\left[\left|\mathcal{F}_{\alpha} u\right|^{2}\right](y) .
\end{aligned}
$$

It follows that the knowledge of $|u(x, t)|$ is equivalent to the knowledge of $A(u)$ on the line $\mathbb{R}(1, t)$. In other words, the Phase Retrieval Problem 1 has been changed into a sampling problem for the ambiguity function. Exploiting this link, we have been able to prove the following:

Theorem II.1 (Jaming [Ja]). Let $u_{0}, v_{0} \in L^{2}(\mathbb{R})$ and $u, v$ be the corresponding solutions of (I.1). Let $\tau \subset \mathbb{R}$ be a set of times and assume that

$$
|u(x, t)|=|v(x, t)| \quad \text { for every } x \in \mathbb{R}^{d} \text { and } t \in \tau \text {. }
$$

1) If $\tau=\mathbb{R}$ (or is dense in $\mathbb{R})$. Then there exists $c \in \mathbb{C}$ with $|c|=1$ such that $v=c u$.

2) If $\tau \subset \mathbb{R}$ has an accumulation point (in particular if $\tau$ has positive measure) and if $u_{0}, v_{0}$ are both compactly supported, then there exists $c \in \mathbb{C}$ with $|c|=1$ such that $v=c u$.

3) Let $a>0$ if $u, v \in L^{2}(\mathbb{R})$ have compact support included in $[-a, a]$ and if $\tau=\left\{\frac{k}{a^{2}}, k \in \mathbb{Z}\right\}$ then there exists $c \in \mathbb{C}$ with $|c|=1$ such that $v=c u$.

Sketch of proof. If $|u(x, t)|=|v(x, t)|$ for every $x, t$ then $A(u)=A(v)$ and it is well known that this implies $u=v$.

The two other items are based on the observation that $A(u)(x, \cdot)$ is the Fourier transform of the function $\varphi_{x}(t):=$ $u\left(t+\frac{x}{2}\right) \overline{u\left(t-\frac{x}{2}\right)}$. But, if $u$ is compactly supported, so is $\varphi_{x}$, thus $A(u)$ is an entire function in the second variable thus if $A(u)(x, \cdot), A(v)(x, \cdot)$ coincide on a large set, they coincide everywhere.

Further, if $u$ and $v$ are supported in intervals, so is $\varphi_{x}$ and we may simply apply Shannon's sampling theorem.

This theorem still requires the knowledge of the solution at infinitely many times. It turns out that if we ask $u, v$ to be "structured", then less is needed:

Theorem II.2 (Jaming [Ja]). Let $u_{0}, v_{0} \in L^{2}(\mathbb{R})$ and $u, v$ be the corresponding solutions of (I.1). Let $\tau \subset \mathbb{R}$ be a set of times and assume that

$$
|u(x, t)|=|v(x, t)| \quad \text { for every } x \in \mathbb{R}^{d} \text { and } t \in \tau \text {. }
$$

1) Assume $u_{0}, v_{0}$ are Hermite functions (of the form $\mathrm{P \gamma}$ where $P$ is a polynomial and $\left.\gamma(x)=e^{-\pi x^{2}}\right), \tau=$ $\{\cot \alpha\}$ with $\alpha \in \mathbb{R} \backslash \mathbb{Q} \pi$. Then $v=c u$ with $|c|=1$.

2) Assume $u_{0}, v_{0}$ are rectangular pulse trains (of the form $\sum_{\text {finite }} c_{j} \mathbf{1}_{[j, j+\eta)}$ with $0<\eta \leq \frac{1}{2}$ and $c_{j}$ a finite sequence of complex numbers) and $\tau=\left\{t_{0}\right\}, t_{0}>0$, then $v=c u$ with $|c|=1$.

3) Assume $u_{0}, v_{0}$ are of the form $\sum_{\text {finite }} c_{i} e^{-\pi\left(t-a_{i}\right)^{2}}, c_{i} \in$ $\mathbb{C}$ and $a_{i} \in \mathbb{R}$, and $\tau=\left\{t_{0}\right\}, t_{0}>0$, then $v=c u$ with $|c|=1$.

Sketch of proof. The proofs of these three facts are algebraic in nature and require to expand $u_{0}, v_{0}$ in some representing system and to identify coefficients. 
For Hermite functions, the problem is best solved for the FrFT and we use the Hermite basis and write $u(t)=\sum_{j=0}^{N} c_{j} H_{j}$, $v(t)=\sum_{j=0}^{M} c_{j} H_{j}$. Then, as is well known,

$$
\mathcal{F}_{\theta}[u](t)=\sum_{k=0}^{N} c_{k} e^{-i k \theta} H_{k}(t)
$$

and a similar expression holds for $\mathcal{F}_{\theta}[v]$. Then $|v|^{2}=|u|^{2}$ and $\left|\mathcal{F}_{\theta}[v]\right|^{2}=\left|\mathcal{F}_{\theta}[u]\right|^{2}$ is equivalent to

$$
\sum_{j, k=1}^{M} d_{j} \overline{d_{k}} H_{j}(t) H_{k}(t)=\sum_{j, k=1}^{N} c_{j} \overline{c_{k}} H_{j}(t) H_{k}(t)
$$

and

$$
\begin{aligned}
\sum_{j, k=1}^{M} d_{j} \overline{d_{k}} e^{i(k-j) \beta} H_{j}(t) & H_{k}(t) \\
& =\sum_{j, k=1}^{N} c_{j} \overline{c_{k}} e^{i(k-j) \beta} H_{j}(t) H_{k}(t)
\end{aligned}
$$

An induction starting from the highest order term then leads to the result.

For the pulse trains, the ambiguity function reformulation is the most convenient. We first introduce the discrete ambiguity function of a sequence $a$ :

$$
\mathcal{A}(a)(k, y)=\sum_{j \in \mathbb{Z}} a_{j} \overline{a_{j-k}} e^{2 i \pi j y} .
$$

Then we write $u(t)=\sum a_{j} \mathbf{1}_{[0, \eta)}(t-j)$ where $a_{j}$ is a finite sequence. A simple commutation shows that, for $x \in$ $\left[j-\frac{1}{2}, j+\frac{1}{2}\right]$

$$
A(u)(x, y)=e^{i \pi j y} \mathcal{A}(a)(j, y) A\left(\mathbf{1}_{[0, \eta)}\right)(x-j, y)
$$

and a similar expression holds for $A(v)$. The part $A\left(\mathbf{1}_{[0, \eta)}\right)(x-j, y)$ being common to $A(u)$ and $A(v)$, the problem reduces to a problem on the discrete part which is again solve by an induction procedure.

The last case is more elaborate and we refer to [Ja].

This theorem mislead us to conjecture that three different times might be sufficient to obtain uniqueness. This was disproved in [CHST] who noticed that as soon as there exists a triplet for which non-uniqueness could be achieved, then no triplet would work and then elaborated on a counter-example given in [Ja] that showed that the condition on the time in the case of Hermite function is necessary. Let us here give a different proof that appeals to an argument of A.E.J.M Janssen [Jan] and an oblique marginal formula for the Zak transform

$$
Z[f](x, \xi)=\sum_{k \in \mathbb{Z}} f(x+k) e^{-2 i \pi k \xi} .
$$

which is of independent interest.
Theorem II.3 (Andreys-Jaming [AJ]).

Let $p \in \mathbb{Z} \backslash\{0\}, q \in \mathbb{N}$ be relatively prime integers and let $\alpha$ be defined by $\cot \alpha=\frac{p}{q}$. For $n \in \mathbb{Z}$, and $x \in[0,1]$, let

$$
c_{n, p, q}=\frac{1}{q} \sum_{k=0}^{q-1}(-1)^{k p} e^{i \pi \frac{p}{q} k^{2}} e^{-2 i \pi \frac{n k}{q}}
$$

and, for $x \in[0,1], n \in \mathbb{Z}$, write $\xi_{n, p, q}(x)=\frac{p}{q} x+\frac{1}{2} p+\frac{n}{q}$ and $A_{p, q}(x)=\left\{n \in \mathbb{Z} \mid 0 \leq \xi_{n, p, q}(x) \leq 1\right\}$. Then, for every $f \in L^{2}(\mathbb{R})$,

$$
\begin{aligned}
& \mathcal{F}_{\alpha} f(\xi)=c_{\alpha} e^{-i \pi \frac{p}{q} \xi^{2}} \int_{0}^{1} e^{-2 i \pi \frac{\xi}{\sin \alpha} x-i \pi \frac{p}{q} x^{2}} \\
& \times \sum_{n \in A_{p, q}(x)} c_{n, p, q} Z f\left(x, \frac{\xi}{\sin \alpha}+\xi_{n, p, q}(x)\right) d x .
\end{aligned}
$$

The identity has to be taken in the $L^{2}(\mathbb{R})$ sense.

This theorem is a (rigourous) elaboration on the formal computation that allows to compute the Zak transform of a chirp. From this, it is easy to obtain the following:

Corollary II.4 (Andreys-Jaming [AJ]). Let $r_{1}, \ldots, r_{m} \in \mathbb{Q}$ be $m$ different rational numbers and $N \in \mathbb{N}$. For $j=1, \ldots, m$ let $\alpha_{j} \in[0, \pi)$ be defined via $\cot \alpha_{j}=r_{j}$. Then there exists $f_{1}, \ldots, f_{N} \in L^{2}(\mathbb{R})$ such that, for $j=1, \ldots, m$ and $k \neq \ell=$ $1, \ldots, N$,

$$
\operatorname{supp} \mathcal{F}_{\alpha_{j}} f_{k} \cap \operatorname{supp} \mathcal{F}_{\alpha_{j}} f_{\ell}=\emptyset .
$$

In particular, for every $j=1, \ldots, m$ and for every $c_{1}, \ldots, c_{N} \in \mathbb{C}$ with $\left|c_{k}\right|=1$,

$$
\left|\mathcal{F}_{\alpha_{j}}\left[\sum_{k=1}^{N} c_{k} f_{k}\right]\right|=\left|\mathcal{F}_{\alpha_{j}}\left[\sum_{k=1}^{N} f_{k}\right]\right| .
$$

Taking $u_{0}=\sum_{k=1}^{N} f_{k}, v_{0}=\sum_{k=1}^{N} c_{k} f_{k}$ and solving the Shrödinger equation with these initial conditions, we obtain functions $u(t, x), v(t, x)$ that have modulus coinciding at $m$ different times but still being different.

Corollary II.4 is established as follows: if one takes a function $F \in L^{2}$ supported in a (union) small disc in $[0,1] \times[0,1]$, then this function is the Zak transform of some function $f \in L^{2}(\mathbb{R}), F=Z f$ and the support of $\mathcal{F}_{\alpha}[f]$ is then obtained from (II.2) by looking at all oblique lines $\left(x, \xi \sin \alpha+\xi_{n, p, q}(x)\right)$ appearing in that formula that intersect the disc (see Figure 1).

\section{The Helmholtz EQUATION}

The aim of this section is to show that solutions of the Helmholtz equation are often uniquely determined by their modulus. The main result is the following:

Theorem III.1 (Jaming \& Pérez-Esteva [JPE]). Let $u_{f}, u_{g} \in$ $\mathcal{W}^{2}\left(\mathbb{R}^{d}\right)$ be such that $\left|u_{g}\right|=\left|u_{f}\right|$. Assume one of the following holds:

- $f$ and $g$ are real valued;

- $f$ has non-zero mean;

- the dimension is $d=2$; 


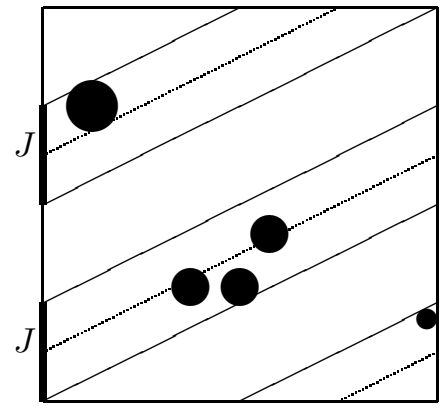

Fig. 1. In this figure $p=2, q=1$ and the slope is $\frac{p}{q}=\frac{1}{2}$. The support of $Z[f]$ is the union of the black discs. Then the set $J$ is such that, for each $\eta \in J$, the (periodic) line starting at $(0, \eta)$ with slope $q / p=1 / 2$ $(\tan \alpha=q / p)$ intersects at least once the support of $Z f$ (one such line is drawn dotted). Here $J=[0,1 / 4] \cup[1 / 2,3 / 4]$ Finally, the support of $\mathcal{F}_{\alpha}[f]$ is then included in the set of all $\xi$ such that $\xi / \sin \alpha+p / 2 \in J+$ $\mathbb{Z}=\bigcup_{k \in \mathbb{Z}}[k / 2,(k+1 / 2) / 2]$. As $p=2, q=1$, sin $\alpha=2 / \sqrt{5}$, thus $\operatorname{supp} \mathcal{F}_{\alpha}[f] \subset \bigcup_{k \in \mathbb{Z}}[k / \sqrt{5},(k+1 / 2) / \sqrt{5}]$.

$-d \geq 3$ and $f, g$ are zonal functions.

Then either $u_{g}=c u_{f}$ or $u_{b}=c \overline{u_{f}}$ for some $c \in \mathbb{C}$ with $|c|=1$.

Recall that a zonal function is a function of the form $\varphi\left(\left\langle x, x_{0}\right\rangle\right)$ for some $x_{0} \in \mathbb{S}^{d-1}$.

Sketch of proof. The case $f, g$ being real is of a slightly different nature to the other statements. In this case, $u_{f}, u_{g}$ are also real and $\left|u_{f}\right|=\left|u_{g}\right|$ is equivalent to $u_{f}^{2}=u_{g}^{2}$ thus $\left(u_{f}-u_{g}\right)\left(u_{f}+u_{g}\right)=0$. It follows that at least one of $u_{f}=u_{g}$ or $u_{f}=-u_{g}$ holds on a set of positive measure. But they are also analytic, so that if such a relation holds on a set of positive measure, it holds everywhere.

Next notice that if $u_{f} \in \mathcal{W}^{2}\left(\mathbb{R}^{d}\right)$, then (I.4) implies

$$
\left|u_{f}(r \theta)\right|^{2}=\frac{2 \pi}{r^{d-2}} \sum_{m, n=0}^{\infty} c_{m, n}(f) J_{m+\frac{d-2}{2}}(r) J_{n+\frac{d-2}{2}}(r)
$$

where

$$
c_{m, n}(f)=\sum_{j=1}^{N(m)} \sum_{k=1}^{N(n)} a_{m, j}(f) \overline{a_{n, k}(f)} Y_{m}^{j}(\theta) \overline{Y_{n}^{k}(\theta)} .
$$

The key observation is that, thanks to the properties of the Bessel functions, $\left|u_{f}\right|=\left|u_{g}\right|$ can be reformulated in terms of the $c_{m, n}$ 's only:

Lemma III.2. Let $u_{f}, u_{g} \in \mathcal{W}^{2}\left(\mathbb{R}^{d}\right)$. Then $\left|u_{f}\right|=\left|u_{g}\right|$ if and only if for every $0 \leq m \leq n$

$$
\Re\left(c_{m, n-m}(f)\right)=\Re\left(c_{m, n-m}(g)\right) .
$$

Then $a_{0,1}(f)$ is the mean of $f$ and $\left|a_{0,1}(f)\right|^{2}=c_{0,0}(f)=$ $c_{0,0}(g)=\left|a_{0,1}(g)\right|^{2}$. Up to replacing $f, g$ by multiples of them, we can assume that $a_{0,1}(f)=a_{0,1}(g)$ is real (and non zero). Then $\Re\left(c_{0, n}(f)\right)=\Re\left(c_{0, n}(g)\right)$ for every $n$.
But, if we chose the basis of spherical harmonics to be real valued, this implies that $\Re\left(a_{j, k}(f)\right)=\Re\left(a_{j, k}(f)\right)$ for every $j, k$ thus $\Re(f)=\Re(g)$ and $\Re\left(u_{f}\right)=\Re\left(u_{g}\right)$. As $\left|u_{g}\right|^{2}=\left|u_{f}\right|^{2}$ we deduce that $\left|\operatorname{Im}\left(u_{g}\right)\right|^{2}=\left|\operatorname{Im}\left(u_{f}\right)\right|^{2}$. But $\operatorname{Im} u_{g}=u_{\operatorname{Im}(g)}, \operatorname{Im} u_{f}=u_{\operatorname{Im}(f)}$ are real Herglotz functions. The real valued case then implies that

$$
\text { either } \operatorname{Im} u_{g}=\operatorname{Im} u_{f} \text { or } \operatorname{Im} u_{g}=-\operatorname{Im} u_{f} \text {, }
$$

that is $g=f$ or $g=\bar{f}$.

The case of zonal functions is of similar flavor when $d \geq 3$.

The $d=2$ case uses the fact that $\Re c_{m, m}(f)=\Re c_{m, m}(g)$ reduces to

$$
\left|\widehat{f}(m) e^{i m \theta}+\widehat{f}(-m) e^{-i m \theta}\right|=\left|\widehat{g}(m) e^{i m \theta}+\widehat{g}(-m) e^{-i m \theta}\right|
$$

so that, for each $m$, there exists $\kappa_{m} \in \mathbb{C}$ with $\left|\kappa_{m}\right|=1$ such that

$$
\begin{aligned}
& \text { - either } \widehat{f}(m) e^{i m \theta}+\widehat{f}(-m) e^{-i m \theta}=\kappa_{m}\left(\widehat{g}(m) e^{i m \theta}+\right. \\
& \left.\widehat{g}(-m) e^{-i m \theta}\right) \\
& \frac{\text { or } \widehat{f}(m) e^{i m \theta}}{\overline{\kappa_{m}\left(\widehat{g}(m) e^{i m \theta}+\widehat{g}(-m) e^{-i m \theta}\right)}}+\widehat{f}(-m) e^{-i m \theta}
\end{aligned}
$$

One then uses the other relations to show that $\kappa_{m}$ does not depend on $m$ and that the choice between both alternatives also does not depend on $m$.

\section{REFERENCES}

[AS] M. Abramowitz \& I. A. Stegun, I A Handbook of Mathematical Functions. Dover, New York, 1972.

[AJ] S. ANDREYS \& PH. JAMING, Zak Transform and non-uniqueness in an extension of Pauli's phase retrieval problem. Analysis Mathematica 42 (2016) 185-201.

[CHST] C. CARmeli, T. HeinosaAri, J. Schultz \& A. Toigo Nonuniqueness of phase retrieval for three fractional Fourier transforms. Applied and Comp. Harm. Anal. 39 (2015) 339-346.

[CSV] E. J. CANDÈs, T. STROHMER AND V. VORONINSKi PhaseLift: exact and stable signal recovery from magnitude measurements via convex programming. Comm. Pure and Appl. Math. 66 (2013) 1241-1274.

[FD] F. FENG \& D. DAI, Approximation Theory and Harmonic Analysis on Spheres and Balls. Springer Monographs in math, 2013, Springer, New York.

[Fi] J. R. FIENUP Phase retrieval algorithms: a comparison. Applied Optics 21 (1982), 2758-2769.

[Hu] N. E. HuRT Phase Retrieval and Zero Crossing (Mathematical Methods in Image Reconstruction). Math. and Its Appl. Kluwer Academic Publisher, 1989.

[Ja] $\mathrm{PH}$. JAMING Uniqueness results in an extension of Pauli's phase retrieval problem. Applied and Comp. Harm. Anal. 37 (2014) 413-441.

[JPE] PH. JAMING \& S. PÉREZ-EsTEVA The phase retrieval problem for solutions of the Helmholtz equation. ArXiv:1703.03742.

[Jan] A.J.E.M. JANSSEN The Zak transform and some counterexamples in time-frequency analysis. IEEE Trans. Inform. Theory 38 (1992) 168171.

[KST] M. V. Klibanov, P. E. Sacks \& A. V. Tikhonravov The phase retrieval problem. Inverse problems 11 (1995), 1-28.

[LBL] D. R. LUKE, J. V. BURKE \& R. G. LYON Optical Wavefront Reconstruction: Theory and Numerical Methods. SIAM Review 44 (2002), 169-224.

[Mi] R. P. Millane Phase retrieval in crystallography and optics. J. Opt. Soc. Am. A. 7 (1990), 394-411.

[St] H. StARK, ED. Image Recovery: Theory and Application. Academic Press, New York, 1987.

[WdAM] I. Waldspurger, A. D’Aspremont \& Mallat Phase Recovery, MaxCut and Complex Semidefinite Programming. Math. Prog. A 149 (2015à, 47-81.

[Wa] G. N. WATSON A treatise on the theory of Bessel functions. Cambridge Univ. Press, Cambridge, 1944. 